\title{
Análise da qualidade dos dados das Fichas de Notificação Compulsória de Dengue e Chikungunya
}

\author{
Analysis of the quality of data from the Compulsory Notification Forms of Dengue and \\ Chikungunya \\ Análisis de calidad de datos de los Papeles de Declaración Obligatoria de Dengue y Chikungunya
}

Recebido: 12/07/2021 | Revisado: 19/07/2021 | Aceito: 21/07/2021 | Publicado: 29/07/2021

Tatiana Feliciano

ORCID: https://orcid.org/0000-0001-8816-1965 Universidade Federal Fluminense, Brasil

E-mail: tatianafeliciano@id.uff.br

Benedito Carlos Cordeiro

ORCID: https://orcid.org/0000-0001-6387-511X Universidade Federal Fluminense, Brasil E-mail: bcordeiro@id.uff.br

\begin{abstract}
Resumo
As fichas de notificação são documentos importantes por conter informações que compõem dados epidemiológicos e estatísticos responsáveis por indicadores de saúde, prevenção e controle de doenças. A Dengue e a Chikungunya têm gerado grandes preocupações em relação à saúde pública mundial, o que reforça e evidencia a importância de ações de ensino na saúde sobre estas doenças. O objetivo deste estudo é analisar a qualidade dos dados das fichas de Notificação Compulsória de Dengue e Chikungunya, visando apresentar estratégias para ações em Educação Permanente em Saúde (EPS). Elegeu-se o método de pesquisa através de estudo quantitativo e documental. A coleta de dados foi realizada em 1.934 Fichas de Notificação Individual (FNI), Fichas de Notificação/Conclusão e Fichas de Investigação de Dengue e Febre Chikungunya, que tiveram seu preenchimento no período de $1^{\circ}$ de janeiro a 31 de dezembro de 2019 em um município da região metropolitana do Rio de Janeiro. A doença mais notificada foi a Chikungunya, presente em $77,5 \%$ das notificações, e o principal profissional notificador é o Enfermeiro, responsável por 43,1\% dos registros. Somente $0,8 \%$ das fichas de notificação são preenchidas de modo completamente correto e, das 71 variáveis pesquisadas, 29 apresentaram falhas no preenchimento. O percentual de registros duplicados é de $12,1 \%$. É real e evidente a necessidade de intervenções que melhorem a qualidade das notificações, assim como a criação de estratégias em EPS, para conscientizar, estimular e dar estrutura para que os profissionais realizem a notificação com maior riqueza e qualidade de dados.
\end{abstract}

Palavras-chave: Notificação compulsória; Dengue; Febre de Chikungunya; Educação continuada.

\begin{abstract}
Notification forms are important documents as they contain information that composes epidemiological and statistical data responsible for indicators of health, prevention and disease control. Dengue and Chikungunya have generated great concerns in relation to public health worldwide, which reinforces and highlights the importance of health education actions about these diseases. The objective of this study is to analyze the data quality of the Compulsory Notification Forms for Dengue and Chikungunya, aiming to present strategies for actions in Permanent Health Education (EPS). The research method was chosen through quantitative and documentary study. Data collection was carried out on 1,934 Individual Notification Forms (FNI), Notification/Conclusion Forms and Investigation Forms for Dengue and Chikungunya Fever, which were filled in from January 1st to December 31st, 2019 in a municipality of the metropolitan region of Rio de Janeiro. The most reported disease was Chikungunya, present in $77.5 \%$ of the notifications, and the main notifying professional is the Nurse, responsible for $43.1 \%$ of the records. Only $0.8 \%$ of the notification forms are filled out completely correctly and, of the 71 variables surveyed, 29 showed failures in filling out. The percentage of duplicate records is $12.1 \%$. The need for interventions to improve the quality of notifications is real and evident, as well as the creation of strategies in EPS, to raise awareness, encourage and provide structure for professionals to perform the notification with greater richness and quality of data.
\end{abstract}

Keywords: Compulsory notification; Dengue; Chikungunya fever; Continuing education.

\section{Resumen}

Los papeles de notificación son documentos importantes por contener informaciones que componen datos epidemiológicos y estadísticos responsables por los indicadores de salud, prevención y control de enfermedades. La Dengue y la Chikungunya tienen generado grandes preocupaciones en relación a la salud pública mundial, lo que 
fortalece y demuestra la importancia de acciones de encino en salud sobre esas enfermedades. El objetivo de este estudio es analizar la calidad de datos de los Papeles de Declaración Obligatoria de Dengue y Chikungunya, pretendiendo presentar estrategias para la Educación Permanente en Salud (EPS).Fue escogido como método de investigación el estudio cuantitativo y documental. La coleta de datos fue realizada en 1.934 Papeles de Notificación Individual (PNI), Papeles de Notificación/Conclusión y Papeles de Investigación de Dengue y Fiebre Chikungunya, llenados en el periodo de 1 de Enero a 31 de Diciembre de 2019 en una ciudad de la región metropolitana de Rio de Janeiro. La enfermedad más declarada fue la Chikungunya, presente en 77,5\% de las declaraciones y el principal profesional declarador es el Enfermero, responsable por 43,1\% de los registros. Solamente $0,8 \%$ de los papeles de declaración son llenados en la más completa corrección y, de las 71 variables investigadas, 29 no fueran llenadas adecuadamente.El porcentual de registros duplicados es $12,1 \%$. Es real y evidente la necesidad de intervenciones que mejoren la calidad de las declaraciones, tal cual la creación de estrategias en EPS, por concienciar, estimular y conferir estructura para que los profesionales realicen la declaración con mayor riqueza y calidad de datos.

Palabras clave: Declaración Obligatoria; Dengue; Fiebre de Chikungunya; Educación Continua.

\section{Introdução}

O processo de trabalho desenvolvido pelos profissionais de saúde sobre a notificação compulsória das doenças, agravos e eventos de saúde pública foi a inspiração para este estudo. A complexidade deste processo instigou a pesquisa sobre a frequência das notificações de Dengue e Chikungunya, a distribuição de itens preenchidos corretamente segundo a unidade notificadora e o profissional notificador e as principais falhas observadas no preenchimento das fichas de notificação.

O registro correto das informações propicia o entendimento de como os agravos/casos ocorrem, para que se intervenha o mais precocemente possível; sendo assim, o tratamento inadequado dos dados, as dificuldades na busca ativa dos casos, a disseminação de doenças, a incontingência de surtos e outros problemas são identificados por meio de instrumentos de coleta de dados da Vigilância Epidemiológica, também através da ficha de notificação. Quando as fichas não são utilizadas corretamente ou são subutilizadas, por ignorar variáveis importantes e registrar informações equivocadas por falta de conhecimento técnico, impedem a atuação efetiva no controle e prevenção de doenças, agravos e eventos de saúde pública (Ferreira, 2018; Souza, 2017).

Através das informações preenchidas pelos profissionais de saúde, as fichas de notificação alimentam o Sistema de Informação de Agravos de Notificação (SINAN). O registro correto das informações destas fichas possibilita inúmeras ações, estratégias e planejamentos em saúde em nível nacional, estadual e municipal, cabendo às autoridades responsáveis pela tomada de decisões medidas de intervenção imediatas, mediatas ou a longo prazo (Brasil, 2007).

Sabe-se que profissionais de saúde ou qualquer cidadão podem comunicar a ocorrência de uma determinada doença, agravou ou evento, sendo necessário um preparo técnico e científico dos profissionais para reconhecerem os casos suspeitos ou confirmados que se tem por obrigação notificar (Brasil, 1975).

As fichas de notificação são documentos de extrema importância para alguns dos indicadores de saúde, pois nelas constam as informações exatas para compor os dados estatísticos que são necessários para traçar metas, estratégias de prevenção e controle de doenças, assim como a provisão e a previsão de materiais e insumos.

Em se tratando de notificações de doenças, as Arboviroses, Dengue e Chikungunya têm gerado grandes preocupações em relação à saúde pública mundial. Transmitidas pelo Aedes aegypti e outros vetores, como o Aedes albopictus, as Arboviroses podem causar até 20 mil mortes anuais, o que reforça a necessidade da manutenção de ações de prevenção, controle, assistência e reabilitação aos infectados, e evidencia a importância de ações de ensino na saúde aos profissionais da área (Brasil, 2019c).

Especialistas da Vigilância Epidemiológica registram objetivos a serem alcançados com as ações estratégicas contra as Arboviroses, dentre elas: a melhoria da qualidade das informações, desestímulo à subnotificação e o fortalecimento das ações de Educação Permanente em Saúde (EPS). Estas estratégias são fundamentais para embasar e solidificar os indicadores epidemiológicos, com o propósito de implementar medidas de saúde pública de prevenção, proteção, controle e tratamento, reduzindo assim as sequelas evitáveis e óbitos relacionados às Arboviroses (Brasil, 2019b). 
Mediante ao exposto e justificando a obrigatoriedade das notificações, a Portaria de Consolidação $\mathrm{n}^{\circ} 4$, de 28 de setembro de 2017, reforça a determinação das notificações semanais para casos agudos de Dengue e Chikungunya, e imediatamente (até 24 horas) para os casos de óbito e casos em áreas sem transmissão para as três esferas de atenção à saúde (Brasil, 2017a).

Esta pesquisa justifica-se pela fundamental necessidade de se garantirem estudos para a implementação de políticas públicas eficientes, visando a melhoria na qualidade dos dados e meios que efetivem o aprimoramento profissional para essa questão (Muguande, 2011).

Deste modo, o objetivo deste estudo é analisar a qualidade dos dados das fichas de Notificação Compulsória de Dengue e Chikungunya de um município da região metropolitana do Rio de Janeiro, visando apresentar estratégias para ações em Educação Permanente em Saúde.

\section{Metodologia}

A construção deste artigo originou-se do projeto de mestrado intitulado "Análise da qualidade dos dados das Fichas de Notificação de Dengue e Chikungunya”.

Para se alcançar o objetivo deste artigo, elegeu-se um estudo quantitativo e documental, pois entende-se que este método contribui para coleta e análise de dados primários, ou seja, dados brutos obtidos para expor o fenômeno a ser estudado. A análise documental das fichas de notificação apresenta melhor representatividade dos dados e evita interferências da vigilância passiva na pesquisa, ou seja, aquela vigilância distante do paciente, porém com menor investimento financeiro. A coleta de dados direto na fonte principal permite uma melhor análise do conteúdo e melhor envolvimento do pesquisador com a pesquisa de fato. $\mathrm{O}$ critério estabelecido para a organização desta pesquisa foi a fonte documental (Andrade et al., 2018; Pimentel, 2001).

A coleta de dados foi realizada de maio de 2020 a fevereiro de 2021, em 1.934 Fichas de Notificação Individual (FNI), Fichas de Notificação/Conclusão e Fichas de Investigação de Dengue e Febre Chikungunya. O levantamento dos dados foi norteado pela seguinte questão: "qual a qualidade dos dados e o grau de completitude das Fichas de Notificação de Dengue e Chikungunya do ano de 2019?”. As fichas de notificação de Dengue e Chikungunya analisadas tiveram seu preenchimento no período de $1^{\circ}$ de janeiro a 31 de dezembro de 2019, em um município da região metropolitana do Rio de Janeiro.

Uma vez que o objetivo é analisar a qualidade das fichas de notificação de Dengue e Chikungunya, elegeram-se para este estudo as seguintes variáveis: doenças notificadas, principais estatísticas de itens preenchidos corretamente das fichas segundo as unidades notificadoras e segundo o profissional notificador, assim como as principais falhas observadas no preenchimento das fichas.

Os dados coletados foram dispostos em planilha do programa Microsoft Office Excel 2007 e analisados com recursos computacionais do programa IBM-SPSS (Statistical for the Social Science), na versão 22.0. Através da análise descritiva, foi possível sintetizar e caracterizar o comportamento das variáveis. A análise inferencial das variáveis possibilitou testes de significância estatística para analisar se são significativas as diferenças encontradas entre distribuições e estatísticas (proporções e médias) de grupos distintos.

Todas as discussões acerca dos testes de significância foram realizadas considerando nível de significância máximo de $5 \%(0,05)$, ou seja, foi adotada a seguinte regra de decisão nos testes estatísticos: rejeição da hipótese nula sempre que o p-valor associado ao teste for menor que 0,05. Detalhes da metodologia proposta de estatística descritiva e inferencial podem ser encontrados em Triola (2008) e Fávero et al. (2009). 
O projeto de pesquisa do qual originou-se este artigo foi submetido e aprovado pelo Comitê de Ética em Pesquisa da Universidade Federal Fluminense, conforme a Resolução do Conselho Nacional de Saúde no 466, de 12 de dezembro de 2012, sob o número CAAE: 29256319.5.0000.5243.

\section{Resultados e Discussão}

\section{Distribuição das doenças notificadas}

Na análise das fichas de notificação de Dengue e Chikungunya do ano de 2019 do município pesquisado, foram analisadas 1.934 fichas através dos campos com critérios de preenchimento obrigatório e essencial, conforme as instruções para preenchimento das fichas de notificação individual e de conclusão do SINAN (Brasil, 2006).

Para esta pesquisa, verificou-se a necessidade de se inserir o campo "notificador", considerado um campo "desejável” para ações futuras relacionadas a estratégias de ensino-aprendizagem para a Educação Permanente em Saúde.

Os resultados deste estudo são baseados nos dados coletados de uma amostra de 1.934 fichas de notificação compulsória de Dengue e Chikungunya do município de estudado. A Tabela 1 mostra a distribuição de frequências das variáveis que caracterizam estas fichas, de modo que as principais frequências (maior frequência e frequências que diferem da maior frequência em no máximo 5\%) são marcadas em negrito.

De acordo com os dados expostos na Tabela 1, verifica-se que a doença mais frequentemente notificada no município foi a Chikungunya, presente em 77,5\% das notificações. Em segundo lugar, a doença mais notificada é a Dengue, presente em $56,8 \%$ das notificações.

Tabela 1: Distribuição de frequências das variáveis que caracterizam as Fichas de Notificação Compulsória.

\begin{tabular}{l|cc}
\hline \multirow{1}{*}{ Variável } & \multicolumn{2}{c}{ Número de Fichas (n=1934) } \\
\cline { 2 - 3 } & F & \\
\hline Doença Notificada & 43 & $2,2 \%$ \\
Não informada & 1 & $0,1 \%$ \\
Arbovirose & 388 & $20,1 \%$ \\
Dengue & $\mathbf{7 9 0}$ & $\mathbf{4 0 , 8 \%}$ \\
Chikungunya & $\mathbf{7 1 0}$ & $\mathbf{3 6 , 7 \%}$ \\
Dengue e Chikungunya & 2 & $0,1 \%$ \\
Dengue, Chikungunya e Zica & & \\
\hline
\end{tabular}

Fonte: Autores.

A Chikungunya e a Dengue têm sua ocorrência geralmente em forma de surto ou epidemia, logo, pelo fator de evolução de gravidade dos casos, recomenda-se que todos os profissionais de saúde envolvidos estejam habilitados e qualificados para proporcionar os cuidados necessários e cabíveis, assim como toda a previsão e provisão de materiais, insumos e equipamentos necessários para o manejo clínico destas doenças (Brasil, 2017b).

A ficha de investigação de Dengue e Febre Chikungunya, assim como todas as outras fichas de notificação, possuem um campo de preenchimento obrigatório para incluir o nome da doença ou agravo, assim como o código relacionado à classificação da doença. Embora seja um campo de extrema relevância para a inserção da notificação no SINAN, foram identificadas 2,2\% das fichas sem a informação da doença ou agravo a ser notificado. Em um estudo sobre a descrição dos registros repetidos no Sistema de Informação de Agravos de Notificação, Silva Júnior e colaboradores (2016) relacionam o 
aumento dos registros de Dengue ao crescimento populacional provocado pela instalação de uma unidade de refino de petróleo na cidade em 2008, o que proporcionou um grande aumento da população em busca de trabalho, renda e moradia.

Duarte (2006) e Pereira e col. (2015) afirmam ser quase universal nas pesquisas sobre este tema a sugestão de melhoria na qualidade de informação das doenças e agravos de notificação. A qualidade insuficiente dos dados, subnotificações, erros e atraso na conclusão dos casos afetam o cumprimento dos indicadores e estratégias da Vigilância Epidemiológica propostas para os serviços de saúde.

\section{Unidades notificadoras}

Nesta etapa do estudo, foi investigado se o percentual de itens preenchidos corretamente estava associado à unidade notificadora. Para tal, foram considerados três grupos de fichas: grupo de fichas notificadas pelo Hospital Municipal, grupo de fichas notificadas pela Unidade de Pronto Atendimento Municipal (UPA) e grupo de fichas notificadas pelo Núcleo de Epidemiologia Hospitalar (NEH). As demais unidades notificadoras não foram consideradas por insuficiente tamanho de amostra para comparação.

A comparação da distribuição do percentual de itens preenchidos corretamente nos três grupos é exibida na Figura 1 e as principais estatísticas da variável nos três grupos são exibidas na Tabela 2. A significância das diferenças observadas nas distribuições dos três grupos foi avaliada pelo Teste de Kruskal Wallis, que resultou num p-valor menor que 0,001, levando à conclusão de que há diferença significativa entre as distribuições dos três grupos, logo, há uma associação significativa entre o percentual de itens preenchidos corretamente e a unidade notificadora.

Figura 1: Boxplot da distribuição do Percentual de Itens Preenchidos Corretamente na Ficha de Notificação Compulsória nos grupos definidos segundo a unidade notificadora.

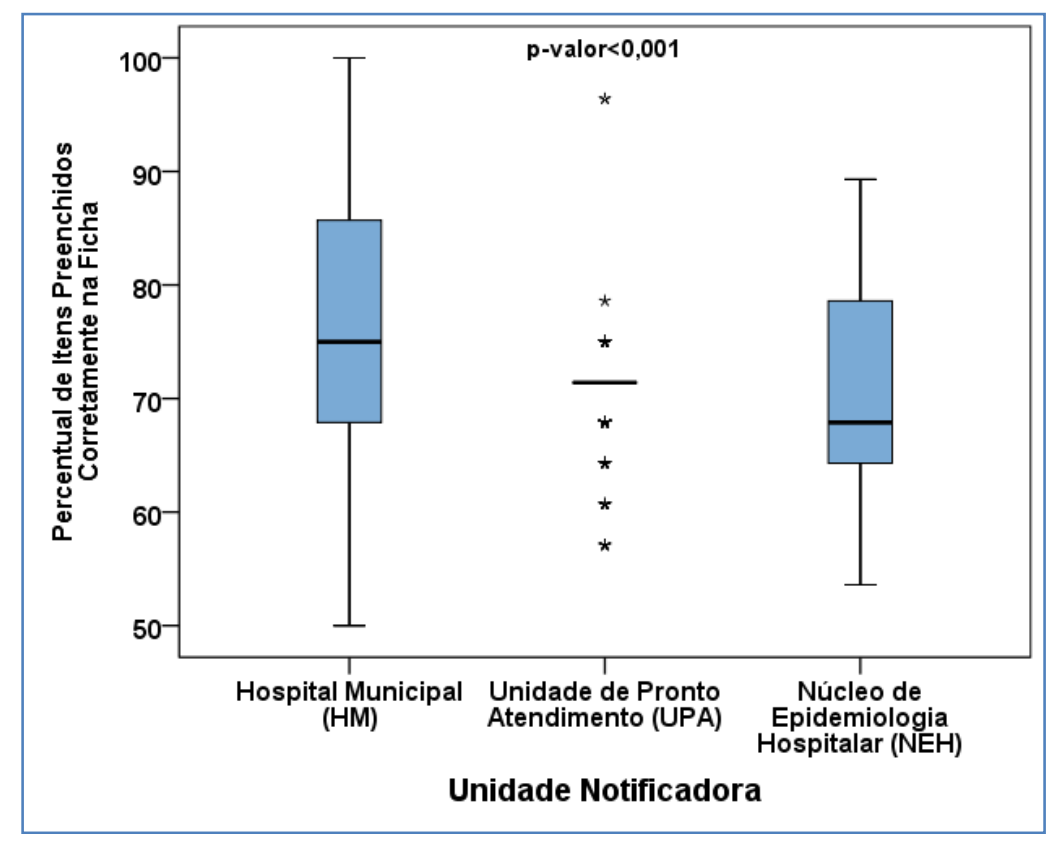

Fonte: Autores.

Em análise post hoc comparando os pares de grupos, observou-se que não há diferença significativa entre as eficiências nos preenchimentos das fichas notificadas na UPA e das fichas notificadas no NEH (p-valor=0,133) e a eficiência no preenchimento das fichas notificadas no HM é significativamente distinta da eficiência no preenchimento das fichas notificadas na UPA e no NEH (p-valores menores que 0,001). 
Tabela 2: Principais estatísticas da distribuição do Percentual de Itens Preenchidos Corretamente na Ficha de Notificação Compulsória nos grupos definidos segundo a unidade notificadora.

\begin{tabular}{l|c|c|c}
\hline atística do Percentual de Itens Preenchidos & \multicolumn{3}{|c}{ Unidade Notificadora } \\
\cline { 2 - 4 } $\begin{array}{l}\text { Corretamente na Ficha de Notificação } \\
\text { Compulsória }\end{array}$ & HM & UPA & NEH \\
\hline Mínimo & 50,0 & 57,1 & 53,6 \\
Máximo & 100,0 & 96,4 & 89,3 \\
Percentil 25 & 67,9 & 71,4 & 64,3 \\
Mediana & $\mathbf{7 5 , 0}$ & $\mathbf{7 1 , 4}$ & $\mathbf{6 7 , 9}$ \\
Percentil 75 & 85,7 & 71,4 & 78,6 \\
Média & $\mathbf{7 7 , 0}$ & $\mathbf{7 0 , 7}$ & $\mathbf{6 9 , 8}$ \\
Desvio Padrão & 10,9 & 3,1 & 9,2 \\
C.V & 0,14 & 0,04 & 0,13 \\
\hline
\end{tabular}

Fonte: Autores.

A unidade notificadora ou o local onde ocorreu o agravo ou evento são campos de preenchimento obrigatório nas fichas de notificação e de grande relevância para o SINAN, para se traçar estratégias a curto, médio e longo prazo (Brasil, 2006).

A maior fonte notificadora foi a unidade hospitalar municipal, o HM, com a média de 77\% das notificações. Esta unidade hospitalar é referência para os atendimentos de urgência e emergência no município.

Vale ressaltar que as informações provenientes da UPA foram preenchidas pelos médicos durante o atendimento em sistema operacional interno com os sinais, sintomas, resultado dos exames laboratoriais e Código Internacional da doença diagnosticada. O responsável pela investigação e coleta dos dados sobre os casos suspeitos de Dengue e Chikungunya através deste sistema foi um profissional da Vigilância Epidemiológica, se baseando nos dados relatados apenas pelos médicos.

As demais unidades notificadoras não consideradas para esta análise por insuficiente tamanho de amostra para a comparação destacam-se neste estudo por haver no município 45 unidades de Atenção Primária à Saúde (APS), mas somente 08 delas notificaram casos de Dengue e Chikungunya em 2019. Decerto, este é um ponto relevante deste estudo que requer atenção e avaliação mais apurada. A baixa frequência de notificações na atenção básica pode estar relacionada a diversos fatores; entretanto, este estudo permite a identificação de uma irregularidade nas notificações e nos remete a refletir sobre o motivo ou a condição que impede a notificação destas doenças estudadas pelos profissionais que atuam na APS deste município.

De fato, Costa e colaboradores (2018) salientam que o planejamento para o controle epidemiológico pode ser afetado pela ausência das notificações, notificação tardia das doenças ou subnotificações. Diante destas circunstâncias, ressalta-se a importância de se implementar ações estratégicas para que estas unidades se reconheçam como fundamentais no Sistema de Vigilância Epidemiológica.

Na perspectiva de auxiliar os profissionais de saúde e os gestores na reorganização dos seus processos de trabalho, a PNEPS aborda a proposta de formular e promover um espaço produtor de dúvidas, alegrias, dor, satisfação, orgulho e sentimentos que reafirmem a relevância do trabalho na APS, segundo Merhy (2016). Desta forma, a EPS visa encontrar as respostas necessárias para a intervenção nos problemas diagnosticados, na tentativa de superar as lacunas existentes no mundo do trabalho.

Da mesma forma como afirmado por Escosteguy e colaboradores (2017) em sua pesquisa, verifica-se a necessidade de reforçar as estratégias de sensibilização e informação nas unidades notificadoras, com o propósito de expor aos profissionais de saúde a relevância das notificações para o Sistema de Vigilância em Saúde. 
Vale ressaltar que os estabelecimentos privados de saúde e de ensino têm o compromisso, de acordo com a Lei $n^{\circ} 6.259$, de 1975, de informar aos gestores do Sistema Único de Saúde o caso, evento ou incidente de notificação compulsória. Ainda assim, têm a permissão para se cadastrar no SINAN como fonte notificadora, a fim de contribuir com dados epidemiológicos (Brasil, 2007).

\section{Percentual de itens preenchidos corretamente segundo o profissional notificador}

Com o propósito de obter dados para futuras propostas e estratégias de EPS, optou-se por analisar a eficiência de itens preenchidos corretamente nas fichas de notificação compulsória nos grupos definidos segundo o profissional notificador, conforme Figura 2.

O Guia de Instruções para o Preenchimento das Fichas de Notificação (Brasil, 2019a) e o dicionário SINAN-net (Brasil, 2006), destacam a importância dos campos obrigatórios e essenciais, almejando uma melhor investigação e a geração de indicadores de saúde que garantam a qualidade dos dados. Optou-se por atribuir o termo "desejável” ao campo "notificador", com o propósito de futuramente ter subsídios para estratégias em ensino na saúde (Abath et al., 2014).

Figura 2: Boxplot da distribuição do Percentual de Itens Preenchidos Corretamente na Ficha de Notificação Compulsória nos grupos definidos segundo o profissional notificador.

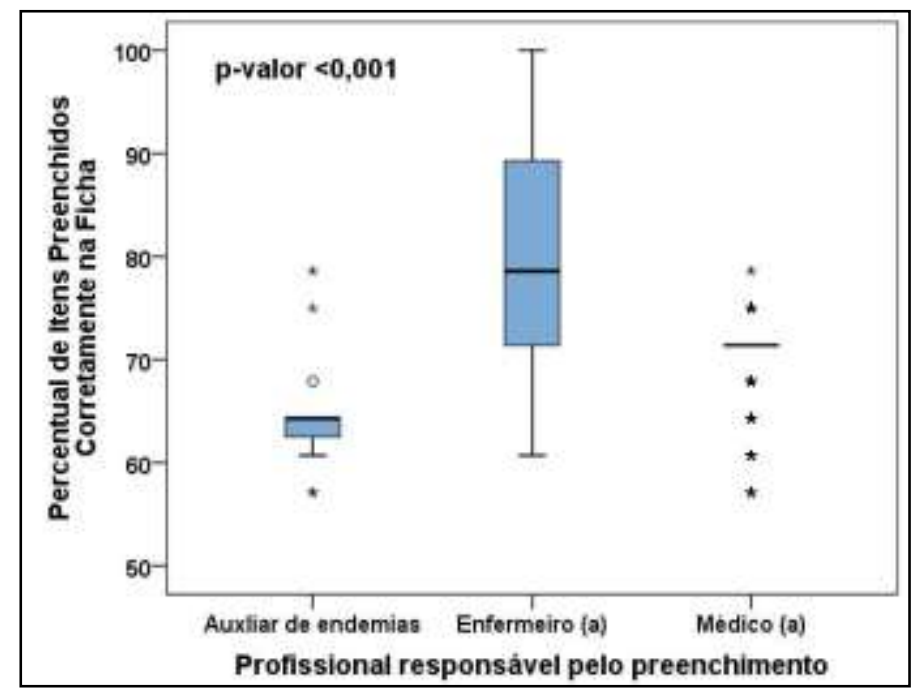

Fonte: Autores.

Em análise post hoc comparando os pares de grupos, observou-se que há diferença significativa entre as eficiências nos preenchimentos das fichas notificadas por Auxiliar de Endemias e das fichas notificadas por Enfermeiro (p-valor $<0,001)$. A eficiência no preenchimento das fichas notificadas por Auxiliar de Endemias é também significativamente distinta da eficiência no preenchimento das fichas notificadas por Médicos (p-valor $<0,001)$ e a eficiência no preenchimento das fichas notificadas por Enfermeiro é também significativamente distinta da eficiência no preenchimento das fichas por Médicos (p-valor=0,001).

Comparando as estatísticas exibidas na Tabela 3, observa-se que a eficiência no preenchimento das fichas registradas é maior quando o registro é realizado por Enfermeiro e menor quando registrada por Auxiliar de Endemias. Todas as fichas com $100 \%$ de itens preenchidos corretamente foram preenchidas por Enfermeiros, enquanto o maior percentual de acerto das fichas preenchidas por Auxiliar de Endemias e Médicos foi de 78,6\%. Logo, os Enfermeiros se destacam como os profissionais que preenchem as fichas de notificação de doença com maior eficiência. 
Tabela 3: Principais estatísticas da distribuição do Percentual de Itens Preenchidos Corretamente na Ficha de Notificação Compulsória nos grupos definidos segundo o profissional notificador.

\begin{tabular}{l|c|c|c}
\hline \multirow{2}{*}{$\begin{array}{c}\text { Estatística do Percentual de Itens } \\
\text { Preenchidos Corretamente na Ficha de } \\
\text { Notificação Compulsória }\end{array}$} & \multicolumn{3}{|c}{ Profissional responsável pela notificação } \\
\cline { 2 - 4 } & $\begin{array}{c}\text { Auxiliar de } \\
\text { Endemias }\end{array}$ & Enfermeiro & Médico \\
\hline Mínimo & 57,1 & 60,7 & 57,1 \\
Máximo & 78,6 & 100,0 & 78,6 \\
Percentil 25 & 60,7 & 71,4 & 71,4 \\
Mediana & $\mathbf{6 4 , 3}$ & $\mathbf{7 8 , 6}$ & $\mathbf{7 1 , 4}$ \\
Percentil 75 & 64,3 & 89,3 & 71,4 \\
Média & $\mathbf{6 4 , 8}$ & $\mathbf{8 0 , 2}$ & $\mathbf{7 0 , 5}$ \\
Desvio Padrão & 5,7 & 10,2 & 2,7 \\
C.V & 0,09 & 0,13 & 0,04 \\
\hline
\end{tabular}

Fonte: Autores.

Todos os dados solicitados no preenchimento da ficha de notificação são de grande relevância para áreas específicas da vigilância epidemiológica, assim como a identificação do responsável pela coleta dos dados. O profissional notificador não informado neste estudo caracteriza-se por rubrica e assinatura ilegíveis, carimbo não identificável e principalmente pela ausência de identificação do notificador na ficha de notificação. Apesar de não ser um dado obrigatório ou essencial no preenchimento das fichas de notificação, este item foi levado em consideração para favorecer futuras estratégias em EPS.

Vale ressaltar o Núcleo de Epidemiologia Hospitalar (NEH), que cumpre a orientação da Portaria n. ${ }^{\circ} 2.529$, de 23 de novembro de 2004, que institui a obrigatoriedade da Vigilância Epidemiológica em nível hospitalar, uma conquista para o fortalecimento das ações em saúde, visto que em muitos locais a cultura de saúde ainda é centrada no ambiente hospitalar (Brasil, 2004).

Uma das atribuições do NEH é a capacitação e atualização dos profissionais da instituição a fim de proporcionar a vigilância e atenção necessária a doenças, agravos e eventos de saúde pública. Além disso, visa contribuir para melhorar as condições de saúde da população e intervir nos processos de disseminação das doenças transmissíveis.

Neste contexto, a partir das fontes primárias de notificação, permite-se identificar as lacunas e locais onde há necessidade de intensificar as ações e estratégias de EPS com o propósito de aprimorar as informações de saúde geradas (Ferreira, 2020).

O elevado número de notificações sem a identificação do notificador pode ser atribuído ao baixo estímulo ou oportunidades não aproveitadas pelo excesso de trabalho para a completitude do preenchimento das fichas de notificação, segundo Assis e colaboradores (2014).

Apesar de ser obrigatória a notificação pelos profissionais de saúde por determinação legal, Freitas (2017) retrata em sua pesquisa o desconhecimento sobre o processo de notificação, visto que profissionais em sua pesquisa consideram a notificação e o acompanhamento do caso como responsabilidade de outros. A Tabela 4 retrata a frequência de notificações segundo o profissional notificador. 
Tabela 4: Distribuição de frequências das variáveis segundo o profissional notificador.

\begin{tabular}{l|cc}
\hline \multirow{2}{*}{ Variável } & Número de Fichas (n=1934) \\
\cline { 2 - 3 } \multicolumn{1}{c|}{ Profissional Notificador } & F & \% \\
Não informado & & $27,4 \%$ \\
Auxiliar de Endemias & 530 & $0,7 \%$ \\
Enfermeiro & 14 & $\mathbf{4 3 , 1 \%}$ \\
Médico & $\mathbf{8 3 3}$ & $28,8 \%$ \\
\hline
\end{tabular}

Fonte: Autores.

O principal profissional notificador é o Enfermeiro, responsável por 43,1\% dos registros, seguido do Médico, responsável por $28,8 \%$ das notificações. A distribuição de frequências de profissional notificador não informado é de $27,4 \%$, um valor expressivo resultante da análise, o que se expressa como um fator preocupante em relação ao objetivo deste estudo. Sobretudo, se torna inviável a identificação do responsável pelo preenchimento da ficha de notificação.

Salienta-se ainda que a Tabela 4 não faz menção a nenhum outro profissional de saúde além dos Enfermeiros, Médicos e Auxiliares de Endemias no campo notificador; logo, a exigência prevista na Lei Federal no 6.259, de 30 de outubro de 1975, sobre a obrigatoriedade da notificação de doenças e agravos não está sendo cumprida (Brasil, 1975).

Diante disso, a Educação Permanente em Saúde e a Vigilância Epidemiológica devem caminhar lado a lado com a meta de ressignificar a relevância das notificações compulsórias junto aos profissionais de saúde.

Logo, quando o responsável pelo preenchimento da ficha não se identifica com nome do notificador, seja por manter o campo em branco, ilegível ou incompleto, são prejudicadas as estratégias para fortalecer e incentivar as notificações compulsórias pelos profissionais, sobretudo o grupo focal para as ações em Educação Permanente em Saúde (Brasil, 2020).

Conforme descrito na pesquisa de Oliveira e col. (2009), as notificações são avaliadas pelos profissionais como uma atividade em segundo plano, o que compromete a vigilância ativa e consequentemente a qualidade das informações.

Diante do exposto, o resultado corrobora a necessidade de se entender a realidade para diagnosticar o motivo pelo qual os profissionais de saúde não estão notificando, proposta essa recomendada pela PNEPS.

Assim como é obrigatória a identificação das doenças, agravos e eventos de saúde pública pelos profissionais de saúde, a notificação destes contribui e auxilia a vigilância epidemiológica nas ações em saúde específicas, sejam elas na prática assistencial ou em relação à Educação Permanente em Saúde dos profissionais envolvidos no ato de notificar (Freitas, 2017).

É relevante destacar que alguns profissionais não possuem o conhecimento e não se sentem responsáveis pelo preenchimento das notificações em sua unidade de trabalho, fato este que torna evidentemente necessária a implementação de atividades e ações contínuas de Educação Permanente em Saúde com ênfase na suspeita, identificação e notificação de doenças, agravos e eventos de saúde pública (Freitas, 2017).

\section{Falhas no preenchimento das fichas de notificação}

A análise da qualidade dos dados das fichas de notificação resultou na identificação de 29 variáveis sobre as falhas no preenchimento da ficha, entretanto, a Figura 3 exibe somente as frequências das principais falhas observadas no preenchimento das fichas compulsórias, presente em mais de $10 \%$ das notificações. 
Dentre as falhas, a ausência de CEP no endereço do paciente, que ocorre em $86,8 \%$ das fichas, e a ausência do critério de confirmação/descarte da doença, que ocorre em 82,0\% das fichas, foram evidentes na análise, assim como a duplicidade de registro, que ocorre em $12,1 \%$ das notificações.

Figura 3: Principais falhas observadas no preenchimento das fichas de notificação compulsória, presentes em mais de $10 \%$ das fichas.

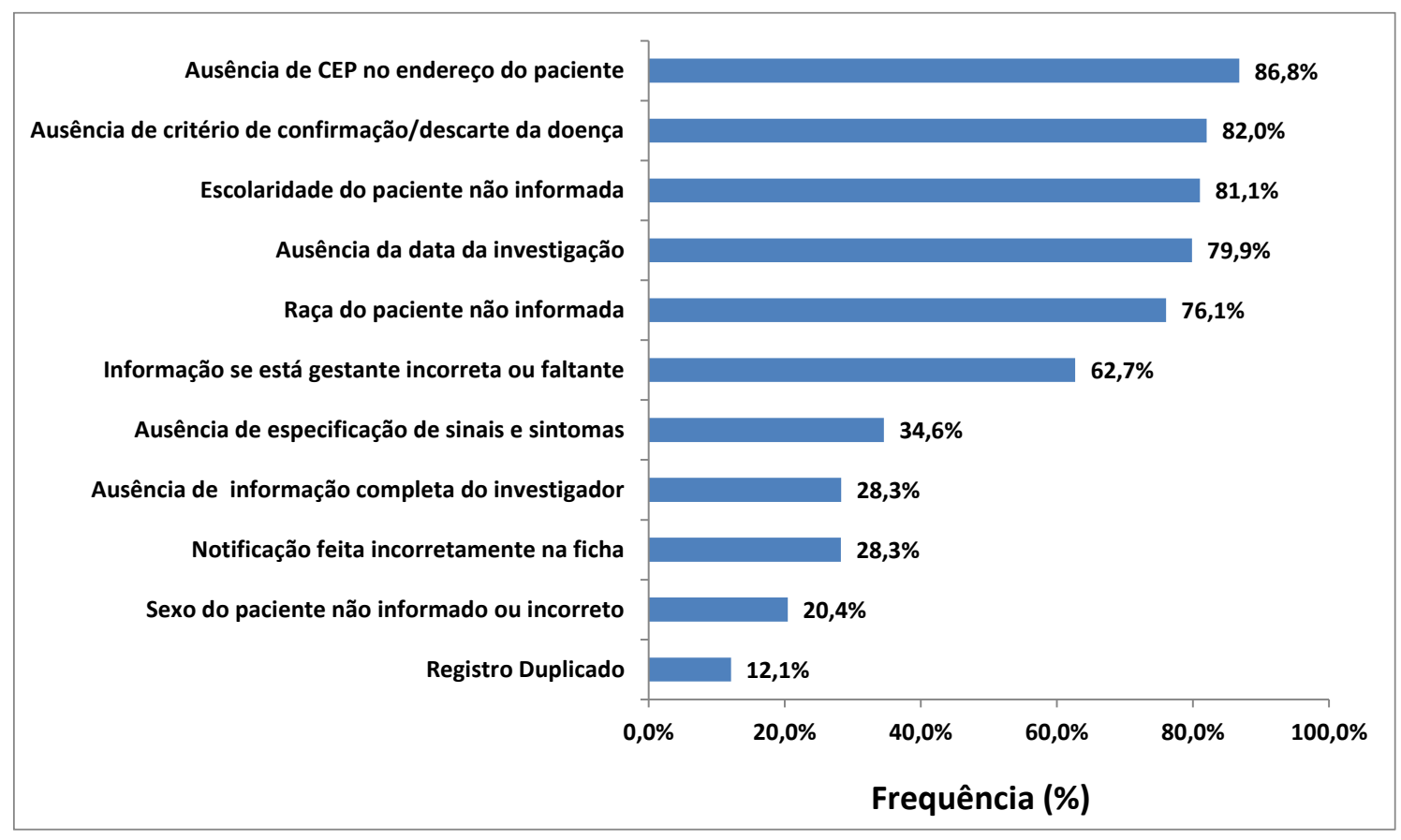

Fonte: Autores.

A partir das ocorrências das falhas descritas na Figura 3, consideradas falhas importantes para a notificação de doenças, foi calculado para cada ficha o percentual de itens preenchidos corretamente, que é uma medida de eficiência do preenchimento da Ficha Compulsória. Somente 0,8\% das fichas de notificação compulsória são preenchidas de modo completamente correto, sem falhas. A maior frequência é de fichas com percentual de itens preenchidos corretamente maior ou igual a $70 \%$ e menor que $80 \%$.

Melo e colaboradores (2018) apresentam fatores que são dificultadores para a realização das notificações, como pouca relevância atribuída a elas pelos profissionais, problemas relacionados ao diagnóstico e registro médico, excesso ou acúmulo de funções a cumprir, a insuficiente habilitação em relação à formação e à qualificação profissional, ou pelo fato dos profissionais não considerarem sua responsabilidade. Estes fatores podem ser observados na Figura 3 através das principais falhas no preenchimento presente em mais de $10 \%$ das fichas.

As variáveis relacionadas à notificação individual e aos dados clínicos das fichas de notificação presentes na Figura 3 representam as maiores ocorrências de falhas. Tal fato pode estar relacionado à vigilância passiva e aos fatores relacionados por Melo e colaboradores (2018) em seu estudo.

As subnotificações, ausência de notificações e duplicidades evidenciadas nesta pesquisa dificultam a identidade epidemiológica em nível local, contribuindo assim para a propagação das doenças passíveis de controle e redução na transmissão, de acordo com Melo e colaboradores (2018).

O termo duplicidade é atribuído à notificação a qual o paciente foi notificado mais de uma vez ou notificado por mais de uma unidade de saúde pela mesma doença/agravo ou evento (BRASIL, 2019). E vale ressaltar que a pesquisa foi realizada 
nas fichas de notificação primárias, ou seja, aquelas preenchidas pelos profissionais de saúde em formulário específico, e a duplicidade foi identificada através do cruzamento dos campos: nome do paciente, data de nascimento e nome da mãe, evitando assim casos homônimos, a duplicidade propriamente dita ou a transferência de local de tratamento seguindo as recomendações do Roteiro para o uso do SINAN Net. (Brasil, 2019a).

O elevado número de duplicidade pode desconfigurar a real identidade epidemiológica de uma determinada localidade e desta forma comprometer a qualidade dos Sistemas de Informação em Saúde (SIS). Este caso é identificado através do percentual de $12,1 \%$ de registros duplicados, conforme Tabela 4.

Se tratando de doenças em que a recuperação é longa, como, por exemplo, a Chikungunya, a duplicidade caracteriza-se fortemente pela busca de atendimento em saúde por mais de uma vez no mesmo estabelecimento de saúde (Silva Júnior, 2016). A Chikungunya, por ter a característica de cronicidade devido aos problemas articulares, é o maior contribuidor para a duplicidade, pois, após a fase aguda da doença, as buscas aos serviços de saúde ainda são frequentes pelas dores articulares.

É recomendado que a notificação de doenças, agravos e eventos de saúde pública obedeçam aos critérios diagnósticos definidos pelo Ministério da Saúde para cada doença descrita no Guia de Vigilância em Saúde, a fim de reduzir as chances de duplicidade e/ou equívoco na notificação (Brasil, 2019b).

À medida que não há um acompanhamento sistemático dessas notificações e, consequentemente, a exclusão das mesmas no sistema, o número de casos de uma determinada doença acaba sendo superestimado, aumentando sua incidência percebida (Brasil, 2007).

De acordo com Honório e colaboradores (2015), são fatores que podem induzir a falhas no preenchimento das fichas de notificação compulsória: a fragilidade e dificuldade de acesso aos diagnósticos laboratoriais, diagnósticos clínicoepidemiológicos pelo conjunto de sinais e sintomas que perpassam entre as doenças, a circulação simultânea dos vírus devido ao clima e condições ideais de criadouro do vetor e a dificuldade na identificação rápida do vírus circulante no momento da epidemia.

Acertadamente Marques e colaboradores (2020) apontam a dificuldade em se estabelecer um diagnóstico rápido, o que reforça a facilidade de adaptação do Aedes, concomitante com as fragilidades nas áreas urbanas e ambientais que propiciam um ambiente favorável para a manutenção e reprodução do vetor.

\section{Considerações Finais}

A análise das fichas de notificação de Dengue e Chikungunya demonstraram que o preenchimento ainda está aquém do desejado. A prevalência de dados obrigatórios, essenciais, ignorados ou em branco é relevante, comprovando indispensável a adoção de medidas para superar estas lacunas.

Fatores identificados nesta pesquisa sugerem que há um hiato entre a formação e a Educação Permanente dos profissionais de saúde, fato para o qual a introdução do capitalismo pode ter contribuído, por a formação do indivíduo estar voltada apenas para o mundo do trabalho, indo na contramão do mundo do ensino, tornando-se notória a dicotomia entre a Formação Profissional e a Educação Permanente (Ceccim, 2005).

É real e evidente a necessidade de intervenções que melhorem a qualidade das notificações de doenças, agravos e eventos de saúde pública, assim como da criação de estratégias em Educação Permanente em Saúde que integrem todos os profissionais que tem a obrigatoriedade de notificação.

Faz parte do Programa Nacional de Educação Permanente em Saúde (PNEPS) promover a aprendizagem significativa, ou seja, fazer com que o profissional já inserido no mercado de trabalho desenvolva atitudes e práticas cognitivas sobre o seu processo de trabalho e não só a repetição de técnicas e procedimentos (Freire, 1989). 
Mediante ao exposto, é necessário conscientizar, estimular e dar estrutura para que os profissionais de saúde realizem as notificações com maior riqueza e qualidade de dados.

O processo de ensino e aprendizagem dos profissionais de saúde deve ser contínuo e permanente, visto que novas demandas surgem a todo momento e requerem esforços dos responsáveis para evitar a queda na qualidade dos dados, subnotificações e duplicidade dos dados (Santos, 2018).

Embora haja uma diretriz legal para a obrigatoriedade de notificação na suspeita ou identificação de doenças, agravos e eventos de saúde pública por parte dos profissionais de saúde, o resultado desta pesquisa destaca a necessidade de novos estudos para superar esta lacuna.

\section{Referências}

Abath, M. B. et al. (2014). Avaliação da completitude, da consistência e da duplicidade de registros de violência do Sinan em Recife, Pernambuco, $2009-2012$. Epidemiologia e Serviços de Saúde, 23(1),131-142. http://scielo.iec.gov.br/scielo.php?script=sci_arttext\&pid=S1679-49742014000100013\&lng=pt\&nrm=iso>.

Andrade, S. R. et al. (2018). Análise documental nas teses de enfermagem: técnica de coleta de dados e métodos de pesquisa. Cogitare Enfermagem, (23)1, e53598. Doi http://dx.doi.org/105380/ce.v23i1.53598.

Assis, V. C.; Amaral, M. P. H. \& Mendonça, A. E. (2014). Análise da qualidade das notificações de dengue informadas no sistema de informação de agravos de notificação, na epidemia de 2010, em uma cidade polo da Zona da Mata do Estado de Minas Gerais. Rev. APS. 17(4), 429-437.

Brasil (1975). Ministério da Saúde. Lei . $^{\circ}$ 6.259, de 30 de outubro de 1975. Dispõe sobre as organizações de Vigilância Epidemiológica, sobre o Programa Nacional de Imunizações, estabelece normas relativas à notificação compulsória de doenças, e dá outras providências.<http://www.portalsinan.saude.gov.br/images/documentos/Legislacoes/Decreto_78231.pdf>

Brasil (2004). Ministério da Saúde. Gabinete do Ministro. Portaria n. ${ }^{\circ}$ 2.529, de 23 de novembro de 2004. Institui o Subsistema Nacional de Vigilância Epidemiológica em Âmbito Hospitalar, integrando o Sistema Nacional de Vigilância Epidemiológica.<http://www.saude.sp.gov.br/resources/cve-centro-devigilancia-epidemiologica/areas-de- vigilancia/nucleo-hospitalar-de-epidemiologia/nhe_portaria25292004.pdf>

Brasil (2006). Ministério da Saúde. Ficha de Notificação - Instruções para Preenchimento. Sinan Net.http://portalsinan.saude.gov.br/images/documentos/Agravos/NINDIV/Notificacao_Individual_v5_instr.pdf>

Brasil (2007). Ministério da Saúde. Secretaria de Vigilância em Saúde. Sistemas de Informações de Agravos de Notificações - Sinan: normas e rotinas. 2ed. http://portalsinan.saude.gov.br/images/documentos/Portarias/Manual_Normas_e_Rotinas.pdf>

Brasil (2017a). Ministério da Saúde. Portaria de Consolidação $n^{\circ} 4$, de 28 de setembro de 2017. Dispõe sobre a consolidação das normas sobre os sistemas e subsistemas do Sistema Único de Saúde.http://bvsms.saude.gov.br/bvs/saudelegis/gm/2017/prc0004_03_10_2017.html>

Brasil (2017b). Ministério da Saúde. Secretaria de Vigilância em Saúde. Chikungunya: manejo clínico. chikungunya_manejo_clinico.pdf (saude.gov.br)>

Brasil (2019a). Ministério da Saúde. Sistema de Vigilância em Saúde. Roteiro para o uso do Sinan Net, análise da qualidade da base de dados e cálculo de indicadores
http://www.portalsinan.saude.gov.br/images/documentosicos

Brasil (2019b). Ministério da Saúde. Secretaria de Vigilância em Saúde. Guia de Vigilância em Saúde: volume único. 3ed.http://portalarquivos2.saude.gov.br/images/pdf/2019/junho/25/guia-vigilancia-saude-volume-unico-3ed.pdf>

Brasil (2019c). Ministério da Saúde. Portal de Boas Práticas em Saúde da Mulher, da Criança e do Adolescente. Arboviroses e gestação: Vigilância em Saúde e Assistência. Postagem em: 13 fev. 2020. https://portaldeboaspraticas.iff.fiocruz.br/atencao-mulher/arboviroses-e-gestacao-vigilancia-em-saude-e-assistencia/>

Brasil (2020). Ministério da Saúde. Sistema de informações de Agravos de Notificação. http://portalsinan.saude.gov.br/notificacoes>

Costa, E. M. S. et al (2018). Avaliação da implantação do Programa de Controle da Dengue em dois municípios fronteiriços do estado de Mato Grosso do Sul, Brasil, 2016. Epidemol. Serv. Saúde. 27(4), e2017478. Doi 10.5123/S1679-49742018000400007>.

Duarte, H. H. P. \& Franca, E. B. (2018). Qualidade dos dados da vigilância epidemiológica da dengue em Belo Horizonte, MG. Rev. Saúde Pública. 40(1), 134142. Documento eletrônico. Doi http://dx.doi.org/10.1590/S0034-8910200600010002>

Favero, L. P. et al (2009). Análise de dados: modelagem multivariada para tomada de decisões. Elsevier.

Ferreira, L. P. et al. (2018). Distúrbio de voz relacionado ao trabalho: proposta de uma ficha de notificação individual. Distúrb. Comum., 30(1), 170-178. 〈https://doi.org/10.23925/2176-2724.2018v30i1p170-178>

Freire, P. (1989). Educação como prática da liberdade. 19ed. Paz e Terra.

Freitas, R. J. M. et al (2017). Atuação dos enfermeiros na identificação e notificação dos casos de violência contra a mulher. HU Revista. 43(2), 91-97. https://periodicos.ufjf.br/index.php/hurevista/article/view/2585/pdf_1>.

Honório, N. A. et al (2015). Chikungunya: uma arbovirose em estabelecimento e expansão no Brasil. Cad. Saúde Pública. 31(5), 906908. <http://www.scielo.br/scielo.php?script=sci_arttext\&pid=S0102-311X2015000500003\&lng=pt\&nrm=iso> 
Research, Society and Development, v. 10, n. 9, e40810918172, 2021

(CC BY 4.0) | ISSN 2525-3409 | DOI: http://dx.doi.org/10.33448/rsd-v10i9.18172

Instituto Brasileiro de Geografia e Estatística (IBGE) (2021). Cidades e Estados. https://www.ibge.gov.br/cidades-e-estados/rj/itaborai.html?>.

Marques, C. A. et al. (2020). Avaliação da não completude das notificações compulsórias de dengue registradas por município de pequeno porte no Brasil. Ciências e Saúde Coletiva. 25(3), 891-900. Doi 10.1590/1413-81232020253.16162018.

Melo, M. A. S. et al. (2018). Percepção dos profissionais de saúde sobre os fatores associados à subnotificação no Sistema Nacional de Agravos de Notificação. Rev. Adm. Saúde.18(71), 1-17.http://dx.doi.org/10.23973/ras.71.104.

Muguande, O. F. et al (2011). Avaliação da qualidade do Sistema de Vigilância Epidemiológica de Doença de Chagas Aguda em Minas Gerais, $2005-2008$. Epidemiol. Serv. Saúde. 20(3), 317-325. Documento eletrônico. Doi 10.5123/S1679-49742011000300006>

Oliveira, M. E. P. et al (2009). Avaliação da completitude dos registros de febre tifoide notificados no SINAN pela Bahia. Epidemiol. Serv. Saúde. 18(3), 219226. Documento eletrônico. http://scielo.iec.gov.br/pdf/ess/v18n3/v18n3a04.pdf>

Pereira, J. C. et al (2015). Perfil e seguimento dos pacientes com tuberculose em município prioritário no Brasil. Rev. Saúde Pública. 49(6), 1-12. Documento eletrônico. http://dx.doi.org/10.1590/S0034-8910.2015049005304>.

Pimentel, A. (2001). O método da análise documental: seu uso numa pesquisa historiográfica. Cadernos de Pesquisa. 114, 183. Documento eletrônico. http://www.scielo.br/pdf/cp/n114/a08n114>

Santos, C. V. B. \& Brandespim, D. F. (2018). Características dos Recursos Humanos e desafios no trabalho de coordenadores de Vigilância em Saúde no interior de Pernambuco. Vigil. sanit. debate. 6(2), 54-60. https://doi.org/10.22239/2317-269x.01027>

Silva Júnior, S. H. A. S. et al. (2016). Descrição dos registros repetidos no Sistema de Informação de Agravos de Notificação, Brasil, 2008-2009. Epidemiol Serv Saúde. 25(3), 487-98. Doi 10.5123/S1679-49742016000300005.

Souza, L. F. M. et al (2017). Análise dos casos de notificação de sífilis congênita em um hospital de referência de Niterói, 2008-2015. DST - Jornal Bras. Doenças Sex. Transm. 29(1), 17-21. <10.5533/DST-2177-8264-201729105>

Triola, M. F. (2018). Introdução à Estatística. 10ed. LTC. 